\title{
Allelopathic potential of 73 weed species in Pakistan
}

Sadiqullah Khan ${ }^{1,2}$, Muhammad Ibrar Shinwari ${ }^{2}$, Khawaja Waqar Ali², Tabassum Rana ${ }^{3}$, Saima Kalsoom ${ }^{3}$ \& Said Akbar Khan ${ }^{4}$

1. Department of Environmental Science, Institute of Environmental Science and Engineering, Gomal University, Dera Ismail Khan, Pakistan; sadiq@iiu.edu.pk

2. Department of Environmental Science, International Islamic University, Sector H-10, Islamabad (44000), Pakistan; m.ibrar@iiiu.edu.pk,khawajawaqar99@gmail.com

3. Sulaiman Bin Abdullah Aba Al-Khail - Centre for Interdisciplinary Research in Basic Science (SA-CIRBS), International Islamic University, Sector H-10, Islamabad (44000), Pakistan; tabassum.sns@gmail.com, genious_chemist@yahoo.com

4. Department of Earth \& Environmental Science, Bahria University, Sector E-8, Islamabad (44000), Pakistan; saidakbar2008@yahoo.com

\section{Received 05-X-2018. Corrected 25-III-2019. Accepted 27-IX-2019.}

\begin{abstract}
Introduction: Use of allelochemicals as inexpensive, biodegradable and eco-friendly substitutes for the management of weeds in crops is a central topic nowadays. Objective: The current study represented the comprehensive screening of allelopathic activity of 73 weeds in Pakistan by the Sandwich method and dish pack method. To investigate the allelochemical compound in top most allelopathic weed. Methods: Allelopathic effects of selected weeds leaves leachate were determined through sandwich technique, while volatile allelopathic effects through dish pack techniques. Qualitative and quantitative phytochemical techniques were applied to investigate allelochemical potential of Melilotus indicus. The statistical analysis of the data described the allelopathic effect of 73 weed plants on lettuce seedling growth in terms of radicle and hypocotyl elongation. Results: Elongation percentage of radicle and hypocotyl ranged $0-74 \%$ to $0-148 \%$ and $0-75 \%$ to $0-84 \%$ respectively at $10 \mathrm{mg}$ concentration of dry plant powder in sandwich method while it was 2-234 \% and 7-150\% at in Dish Pack method. Among the plants screened for phytotoxic activity, M. indicus contained the strongest allelochemicals. Qualitative and quantitative analysis of $M$. indicus showed the presence of flavonoids and phenolic compound along with other allelochemical. Conclusions: Selected weeds may have strong allelochemical potential that can help in the development of bioactive compounds from plant species to be used as natural herbicides and pesticides for sustainable management of weeds and pest.
\end{abstract}

Key words: bioassay, phytochemicals, allelopathy, sandwich method, dish pack method.

Khan, S., Shinwari, M. I., Ali, K. W., Rana, T., Kalsoom, S., \& Khan, S. A. (2019). Allelopathic potential of 73 weed species in Pakistan. Revista de Biología Tropical, 67(6), 1418-1430.

In Pakistan, the wheat crop is facing competition for nutrition and space with some major weeds like Chenopodium album, Avena fatua, Euphorbia helioscopia, Rumex dentatus and Phalaris minor (Ali, Tahir, Shah, Khan, \& Mehmood, 2017). An annual loss of 28 billion Pakistani Rupees is estimated due to weed infestation in wheat crop in Pakistan
(Khan et al., 2018). To supplement the nutrient requirements and weed control, heavy doses of synthetic chemicals (herbicides) are used. However, resistance is developing in different weeds by continuous application of huge quantities of synthetic chemicals that are also a serious threat to the environment. To avoid crop loss by weeds and assure a healthy 
environment, organic weed control measures must be adopted (Fisher et al., 2012; Khan et al., 2018). The demand for organic products has been increased during the last decades (Anwar et al., 2019).

The growth and development of plants are affected by allelochemicals. Allelochemicals isolated from plants could substitute synthetic agrochemicals (Bhadoria, 2011; Appiah et al., 2015). In crop production, weed management through allelopathy is beneficial and environmentally friendly. Allelochemicals have shorter half-lives with different chemical structures and diverse modes of action (Appiah et al., 2015; Abbas et al., 2018). A diverse flora of about 6000 vascular plants including weeds of agro-ecosystem are reported from Pakistan (Shinwari, Shinwari, \& Fujii, 2013). Various allelochemicals are being released to the environment by these plants. The investigation and exploration of these bio-compounds can be carried out for natural herbicides to increase crop yield as well as avoid negative impacts of synthetic chemicals on the environment. The current research focused on screening of allelopathic/herbicidal potential in 73 selected plants for natural weed control strategy through allelopathy.

\section{MATERIALS AND METHODS}

Collection of plant materials: The fresh leaves of 73 weeds were collected from fields, roadsides, wastelands and meadows of Pakistan which were later dried in oven (Biobase) at $45{ }^{\circ} \mathrm{C}$ for approximately $24 \mathrm{~h}$. A milling machine was used for grinding dried leaves in powder form.

\section{Allelopathic screening of selected medic-}

inal weeds: Sandwich and Dish pack screening methods were applied to determine the allelopathic potential of selected medicinal weeds

Sandwich method: For screening the allelopathic potential of selected plant's leaves leachates, the sandwich method was adopted (Fujii et al., 2004) with lettuce (Lactuca sativa) seeds because it is reported earlier that lettuce seeds are reliable for germination and susceptible to chemicals. Agar growth medium is best for seedling germination of lettuce seeds (Shinwari et al., 2013). Growth medium of $7.5 \mathrm{~g}$ agar per $1000 \mathrm{~mL}$ of distilled water was prepared. Selected weed species were screened out in three replicates and mean of the replications ( \pm $\mathrm{SE})$ was presented in data. The untreated agar in multi-well without plant materials was set as control. The multi-wells were sealed airtight with plastic tap and wrapped in aluminum foil. Then multi-well plates were placed in an incubator (Biobase Model BJPX-HI10) for $72 \mathrm{~h}$ at $25{ }^{\circ} \mathrm{C}$. The measurement of radicle and hypocotyl were taken with the help of tweezers and graph papers. Percentage inhibition of radicle and hypocotyl were calculated as:

$$
\% \text { Inhibition }=\frac{(\text { Average length of treatment radicle } / \text { hypocotyl })}{(\text { Average length of control radicle/hypocotyl })}
$$

Dish pack method: For screening the allelopathic potential of selected plants, dish pack method was adopted on lettuce seeds (Fujii, 2005). Multi-dish plastic plates (6 well; $36 \times 18 \mathrm{~mm}$ each) were used for analysis. The distances from the center of the source well (where plant sample was placed) to the center of other wells were 41, 58, 82, and $92 \mathrm{~mm}$ respectively. Oven-dried leaf litter $(100 \mathrm{mg})$ was placed in the source well while in other wells of multi-dish, filter papers were laid and $7 \mathrm{~mL}$ distilled water was added to each well. A respective control was maintained without plant material in the source well. Seven seeds of lettuce were placed on the filter paper of each well. The multi-wells placed were sealed airtight with cellophane tape and wrapped in aluminum foil. The dishes were then placed in an incubator at $25{ }^{\circ} \mathrm{C}$ (Biobase Model BJPXHI10) for three days. The length of radicle and 
hypocotyl were measured and compared to control to show the degree of inhibition.

The experiment was designed using three randomized replicates and the mean of them was reported. In both Dish pack and Sandwich methods for each plant species; the mean and standard deviation (SD) and the standard deviation variance (SDV) was calculated to determine the inhibition pattern of radicles and hypocotyl of the lettuce seedlings (Shinwari \& Fujii, 2013).

Qualitative analysis: Qualitative compound analysis of top allelopathic weed Melilotus indicus crude methanolic extract was carried out for the identification of different allelochemicals classes.

Phenols assessment: The formerly described technique (Richardson, 1990; Majid et al., 2015) was followed for the identification of phenols in $M$. indicus. Each sample (1 $\mathrm{mg}$ ) was suspended in $2 \mathrm{ml}$ of distilled water containing $10 \%$ ferric chloride. Change of suspension to blue or green color showed the presence of phenol.

Flavonoids assessment: For identification of flavonoids in the selected plants samples, the Trease and Evans (1989) protocols were adopted. Briefly, $1 \mathrm{ml}$ of $2 \mathrm{~N}$ sodium hydroxide reacted with $1 \mathrm{mg}$ of sample. A color change to yellow confirmed the presence of flavonoid in M. indicus.

Coumarins assessment: An aliquot sample $1 \mathrm{mg} / \mathrm{ml}$ and $1 \mathrm{ml}$ of $10 \% \mathrm{NaOH}$ was mixed. The appearance of yellow color as a result of a reaction in the test tube indicated coumarins in the test sample (Richardson, 1990).

Saponins assessment: The suspension was formed by mixing of $2 \mathrm{mg}$ of sample in $2 \mathrm{ml}$ distilled water upon vigorous shaking. Formation of a soapy layer of almost $1-2 \mathrm{~cm}$ indicated the presence of saponins (Richardson, 1990).
Tannins assessment: Mixing of $1 \mathrm{mg}$ sample with $2 \mathrm{ml}$ of $5 \%$ ferric chloride gave dark blue or greenish black color which is the confirmative sign of tannins (Trease \& Evans, 1989).

Terpenoids assessment: $0.5 \mathrm{mg}$ of sample was added in $2 \mathrm{ml}$ of each chloroform and concentrated sulphuric acid that formed the red-brown layer and confirmed the presence of terpenoids (Trease \& Evans, 1989).

Anthraquinone assessment: $1 \mathrm{mg}$ sample was mixed with $2 \mathrm{ml}$ of diluted $2 \%$ hydrochloric acid, red color was developed that confirmed anthraquinones (Richardson, 1990).

Anthocyanin and betacyanin assessment: $1 \mathrm{mg}$ plant sample was dissolved in 2 $\mathrm{ml}$ of $01 \mathrm{~N} \mathrm{NaOH}$ and boiled for $10 \mathrm{~min}$. The formation of bluish-green color indicated the anthocyanin and yellow color showed betacyanin presence (Trease \& Evans, 1989).

Alkaloids assessment: The reaction of concentrated Sulphuric acid and $2 \mathrm{mg}$ of the selected sample with Mayer's reagent showed green color or white precipitates that confirmed the presence of alkaloid (Trease \& Evans, 1989).

Total phenolic contents (TPC): Total phenolic contents were determined by spectrophotometer (Kim, Jeong, \& Lee, 2003). The sample was prepared in a volumetric bottle (25 $\mathrm{ml}$ ) by adding $1 \mathrm{ml}$ plant content in $9 \mathrm{ml}$ of deionized water followed by the addition of 1 $\mathrm{ml}$ phenol, and $10 \mathrm{ml}$ of $7 \%$ sodium carbonate and pure deionized water until the final volume of $25 \mathrm{ml}$. The optical density was measured at a wavelength of $750 \mathrm{~nm}$ using Gallic acid as a standard.

Total flavonoid contents (TFC): The spectrophotometric technique was applied to quantify total flavonoid content (Park et al., 2008). The reaction mixture was prepared 
in a test tube containing $0.3 \mathrm{~mL}$ plant content, $0.15 \mathrm{~mL}$ of $0.5 \mathrm{~mol} / \mathrm{L} \mathrm{NaNO}_{2}, 0.3 \mathrm{M} \mathrm{AlCl}_{3}$. $6 \mathrm{H}_{2} \mathrm{O}$ and $3.4 \mathrm{ml}$ of $30 \%$ methanol. After $5 \mathrm{~min}, 1 \mathrm{ml}$ of $\mathrm{NaOH}$ was added and optical density was measured at $506 \mathrm{~nm}$ using rutin standard.

\section{RESULTS}

A total of 73 plant species were studied for their allelopathic potential. Using the sandwich method, Melilotus indicus was identified as the strongest allelopathic plant among the 73 weed species, followed by Medicago parviflora. M. indicus showed strong inhibition on hypocotyl and radicle elongation of lettuce seeds (Fig. 1). The statistical analysis of the Sandwich data is represented in table 1, which described the allelopathic effect of leachates of 73 weeds plants on lettuce seedling elongation (radicle and hypocotyl percentage elongation). It is evident that elongation percentage of radicle and hypocotyl ranged $0-74 \%$ and $0-148 \%$ (10 mg) respectively in Sandwich

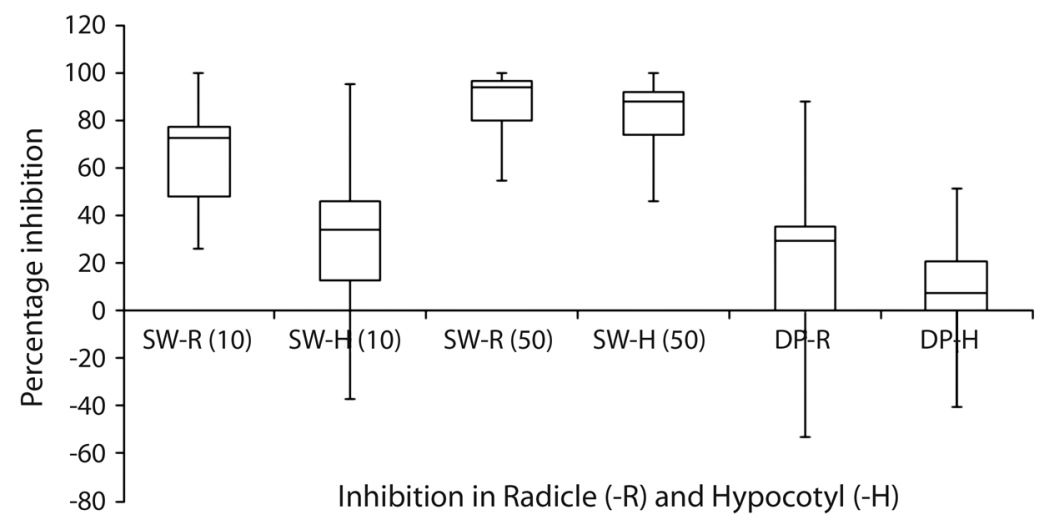

Fig. 1. Range of Percentage Inhibition in Radicle (-R) and Hypocotyl (-H) of 73 Weed Species by Sandwich (SW).

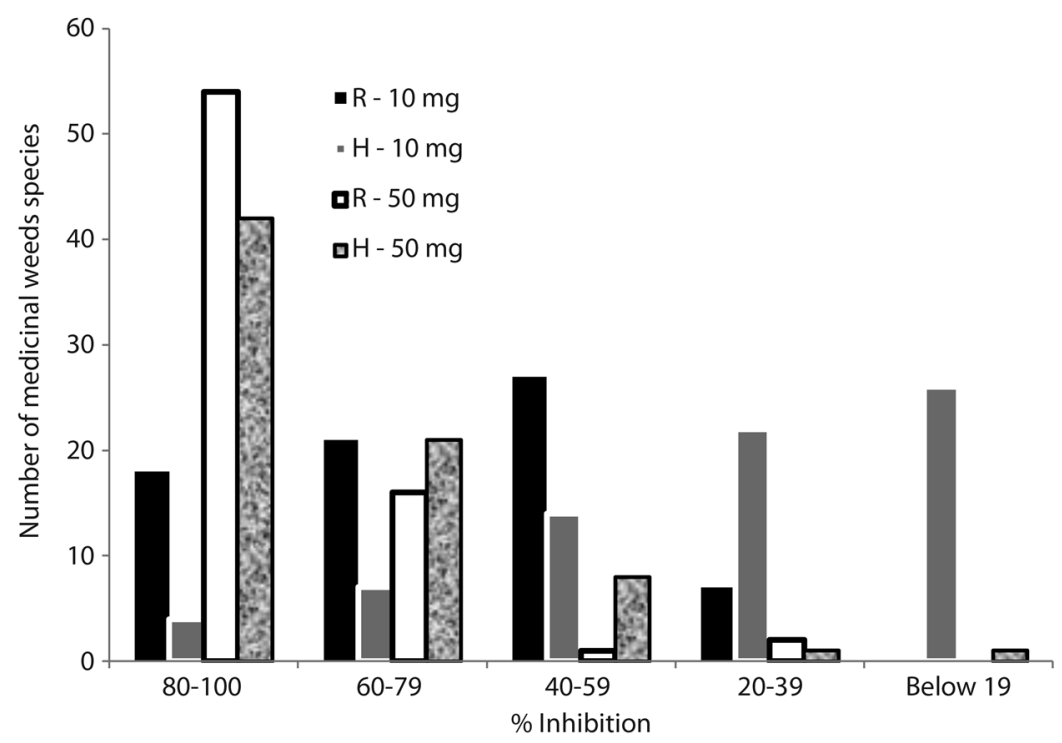

Fig. 2. Frequency Distribution of Percentage Inhibition among medicinal weeds through sandwich method. 


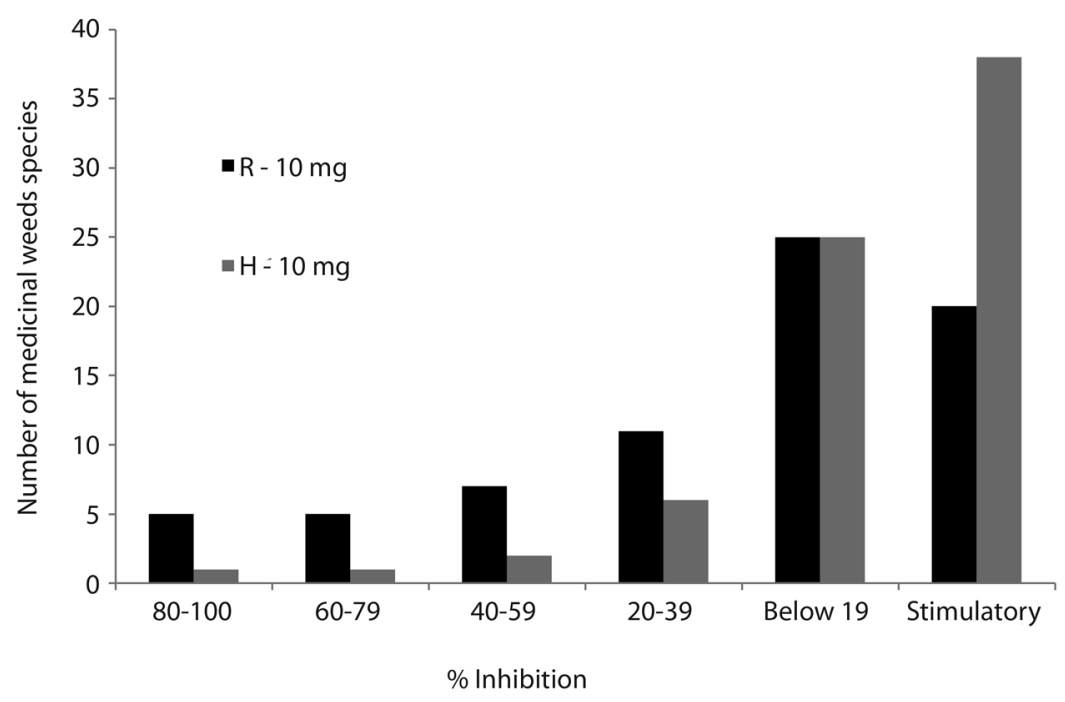

Fig. 3. Frequency distribution of percentage inhibition among medicinal weeds through dish pack method.

method (Table 1, Fig. 2) while 2-234 \% and 7-150 \% in Dish Pack method (Table 2, Fig. 3) compared to control. Sandwich method data of inhibition of radicle at $10 \mathrm{mg}$ concentration is categorized into 5 classes (Table 1). Complete inhibition in the first group (80-100\%) was shown by Melilotus indicus and Medicago parviflora. Strongest inhibitory activity i.e., 90-99 \% was observed in 4 species (Melilotus alba, Peganum harmala, Coronopus didymus and Nasturtium officinale). 80-86 \% inhibition was exhibited by Taverniera cuneifolia, Crotalaria medicaginea, Solanum nigrum, Urtica dioica, Achyranthes aspera, Coriandrum sativum, Otostegia limbata, Avena fatua, Parthenium hysterophorus, Cirsium arvense, Xanthium strumarium, and Anagallis arvensis. The lowest inhibitory activity in this study was observed sizes in the range of $20-39 \%$ by Helianthus annuus, Lythrum salicaria, Dichanthium annulatum, Salvia moocroftiana, Phalaris minor, Cyperus sp., and Saxifraga rotundifolia. Members of Papilionaceae were ranked the strongest inhibitory plant species among the evaluated samples using the sandwich method. Overall there were 8 species from papilionaceae out of which 5 exhibited $80-100 \%$ inhibition while 3 others showed $60-79 \%$ inhibition of radicle growth (Table 1).
In dish pack method (Table 2), Coronopus didymus ranked at the top with $98 \%$ inhibition rate of radical. Next 4 plants with strong inhibition are in the range of 86-93\% i.e., Melilotus alba, Melilotus indicus, Malva parviflora and Solanum nigrum, Nasturtium officinale, Urtica dioica, Cyperus sp., Vicia sativa, Medicago parviflora, comes next in the ranking showing $60-79 \%$ inhibition in radical growth rate. Achyranthes aspera, Crotalaria medicaginea, Amaranthus viridis, Xanthium strumarium, Phalaris minor, Avena fatua, Oxalis corymbos showed $40-59 \%$. A total of 39 plant species showed relatively weak inhibitory potential against the control plant species. 20 plant species exhibited stimulatory potential i.e., Verbena tenuisecta, Saxifraga rotundifolia, Salvia aegyptiaca, Otostegia limbata, Sida alba, Peganum harmala, Oxalis corniculata, Euphorbia hirta, Rumex nepalensis, Adiantum caperis-veneris, Typha minima, Oenothera rosea, Solanum erianthum, Potamogeton lucens, Lythrum salicaria, Cissampelos pareira, Aloe vera, Anisomeles indica, Euphorbia helioscopia and Vernonia anthelmintica. Euphorbia helioscopia and Vernonia anthelmintica remain conspicuous by having more than $200 \%$ elongation rate.

Total phenolic and flavonoid content were identified from the methanolic extract 
TABLE 1

Evaluation of allelopathic activity by leaf litter of 73 medicinal weeds species through Sandwich method at the litter concentration of $10 \mathrm{mg}$

\begin{tabular}{|c|c|c|c|}
\hline \multirow{2}{*}{ Botanical Name } & \multirow{2}{*}{ Family Name } & \multicolumn{2}{|c|}{ Growth } \\
\hline & & Radicle & Hypocotyl \\
\hline \multicolumn{2}{|c|}{ Control } & 100.00 & 100.00 \\
\hline Melilotus indicus L. & Papilionaceae & 0.00 & $0.00 * *$ \\
\hline Medicago parviflora E.H.L. & Papilionaceae & 0.00 & $0.00 * *$ \\
\hline Melilotus alba Desr. & Papilionaceae & 0.82 & 3.41 \\
\hline Peganum harmala $\mathrm{L}$. & Nitrariaceae & 5.05 & 30.61 \\
\hline Coronopus didymus (L.) & Brassicaceae & 6.57 & 19.39 \\
\hline Nasturtium officinale W.T. & Brassicaceae & 7.58 & 24.49 \\
\hline Anagallis arvensis $\mathrm{L}$. & Primulaceae & 9.09 & 33.67 \\
\hline Taverniera cuneifolia Roth. & Papilionaceae & 13.70 & 33.77 \\
\hline Crotalaria medicaginea Lamk. & Papilionacaea & 13.73 & 35.92 \\
\hline Solanum nigrum $\mathrm{L}$. & Solanaceae & 14.14 & 45.92 \\
\hline Urtica dioica $\mathrm{L}$. & Urticaceae & 15.15 & 48.98 \\
\hline Achyranthes aspera $\mathrm{L}$. & Amaranthaceae & 15.66 & 41.84 \\
\hline Coriandrum sativum L. & Apiaceae & 16.33 & 51.13 \\
\hline Otostegia limbata Benth. & Lamiaceae & 17.16 & 55.34 \\
\hline Avena fatua $\mathrm{L}$. & Poaceae & 18.03 & 80.67 \\
\hline Parthenium hysterophorus L. & Asteraceae & 18.11 & 29.55 \\
\hline Cirsium arvense (L.) & Asteraceae & 18.58 & 82.35 \\
\hline Xanthium strumarium L. & Asteraceae & 19.70 & 50.00 \\
\hline Centaurea iberica $\mathrm{T}$. & Asteraceae & 22.73 & 45.92 \\
\hline Trichodesma indicum (L.) & Boraginaceae & 24.04 & 67.23 \\
\hline Digera muricata (L.) & Amaranthaceae & 24.51 & 70.87 \\
\hline Solanum erianthum D Don. & Solanaceae & 24.81 & 54.30 \\
\hline Helianthus annuus L. (petals) & Asteraceae & 25.98 & 66.02 \\
\hline Typha minima Funck. & Typhaceae & 27.27 & 42.86 \\
\hline Potamogeton lucens L. & Potamogetonaceae & 27.40 & 28.57 \\
\hline Malva parviflora $\mathrm{L}$. & Malvaceae & 27.76 & 57.14 \\
\hline Verbena tenuisecta Briq. & Verbenaceae & 27.78 & 55.10 \\
\hline Cyperus sp. & Cyperaceae & 28.79 & 69.39 \\
\hline Argyrolobium roseum Camb. & Papilionaceae & 29.09 & 63.10 \\
\hline Euphorbia hirta L. & Euphorbiaceae & 30.13 & 71.92 \\
\hline Oxalis corniculata L. & Oxalidaceae & 30.81 & 66.33 \\
\hline Sonchus asper (L.) & Asteraceae & 33.74 & 67.05 \\
\hline Pteris cretica $\mathrm{L}$. & Pteridaceae & 34.31 & 60.19 \\
\hline Artemisia scoparia $\mathrm{W} \& \mathrm{~K}$. & Asteraceae & 34.80 & 57.28 \\
\hline Oxalis corymbosa DC. & Oxalidaceae & 35.35 & 77.55 \\
\hline Cannabis sativa $\mathrm{L}$. & Cannabaceae & 36.07 & 90.76 \\
\hline Vicia sativa $\mathrm{L}$. & Papilionacaea & 36.21 & 56.82 \\
\hline Saussurea heteromalla D.Don. & Asteraceae & 38.37 & 78.20 \\
\hline Rhynchosia minima (L.) & Papilionaceae & 39.71 & 85.44 \\
\hline Oenothera rosea L' Her. & Onagraceae & 41.56 & 77.27 \\
\hline Sida cordata (Burm.f.) & Malvaceae & 42.16 & 87.38 \\
\hline Barleria cristata $\mathrm{L}$. & Acanthaceae & 42.82 & 81.05 \\
\hline Convolvulus arvensis $\mathrm{L}$. & Convolvulaceae & 44.36 & 82.12 \\
\hline
\end{tabular}


TABLE 1 (Continued)

\begin{tabular}{|c|c|c|c|}
\hline \multirow{2}{*}{ Botanical Name } & \multirow{2}{*}{ Family Name } & \multicolumn{2}{|c|}{ Growth } \\
\hline & & Radicle & Hypocotyl \\
\hline Taraxacum officinale L. & Asteraceae & 44.44 & 67.05 \\
\hline Ipomoea cornea Mart. & Convolvulaceae & 45.71 & 102.26 \\
\hline Phalaris aquatica $\mathrm{L}$. & Poaceae & 45.86 & 75.50 \\
\hline Commelina benghalensis L. & Commelinaceae & 46.58 & 76.62 \\
\hline Adiantum caperis -veneris L. & Pteridaceae & 47.88 & 79.76 \\
\hline Vernonia anthelmintica $\mathrm{L}$. & Asteraceae & 50.21 & 76.14 \\
\hline Plantago lanceolata $\mathrm{L}$. & Plantaginaceae & 50.23 & 79.22 \\
\hline Chenopodium ambrosioides Briq. & Chenopodiaceae & 50.27 & 87.39 \\
\hline Carthamus oxyacantha M. & Asteraceae & 50.61 & 76.69 \\
\hline Amaranthus viridis $\mathrm{L}$. & Amaranthaceae & 50.75 & 90.07 \\
\hline Salvia aegyptiaca $\mathrm{L}$. & Lamiaceae & 50.91 & 94.05 \\
\hline Lantana camara $\mathrm{L}$. & Verbenaceae & 51.85 & 84.09 \\
\hline Sida alba L. & Malvaceae & 51.88 & 94.04 \\
\hline Conyza bonariensis (L.) & Asteraceae & 52.63 & 78.15 \\
\hline Rumex nepalensis Spreng. & Polygonaceae & 54.32 & 88.64 \\
\hline Nerium oleander L. & Apocynaceae & 54.55 & 92.86 \\
\hline Scrophularia altaica Murray. & Scrophulariaceae & 54.79 & 57.14 \\
\hline Euphorbia helioscopia L. & Euphorbiaceae & 56.33 & 93.23 \\
\hline Cissampelos pareira $\mathrm{L}$. & Menispermaceae & 56.36 & 111.90 \\
\hline Aloe vera (L.) & Asphodelaceae & 57.58 & 88.10 \\
\hline Micromeria biflora Buch. & Lamiaceae & 57.58 & 90.48 \\
\hline Anisomeles indica (L.) & Lamiaceae & 58.18 & 111.90 \\
\hline Pentanema divaricatum Cass. & Asteraceae & 59.48 & 73.06 \\
\hline Helianthus annuus L. (Sepals) & Asteraceae & 61.76 & 105.83 \\
\hline Lythrum salicaria $\mathrm{L}$. & Lythraceae & 62.86 & 148.87 \\
\hline Dichanthium annulatum Forssk. & Poaceae & 67.41 & 89.04 \\
\hline Salvia moocroftiana Wall. & Lamiaceae & 68.42 & 86.09 \\
\hline Phalaris minor Retz. & Poaceae & 69.92 & 101.99 \\
\hline Cyperus rotundus $\mathrm{L}$. & Cyperaceae & 71.84 & 99.25 \\
\hline Saxifraga rotundifolia $\mathrm{L}$. & Saxifragaceae & 74.29 & 99.25 \\
\hline Results are significant at $\mathrm{P}<0.05$ & & & \\
\hline
\end{tabular}

TABLE 2

Evaluation of allelopathic activity by leaf litter of 73 medicinal weeds species through Dish pack method at the litter concentration of $100 \mathrm{mg}$

\begin{tabular}{lccc}
\multicolumn{1}{c}{ Species } & \multicolumn{2}{c}{ Extension \% } & Criteria \\
Coronopus didymus (L.) & Radicle & Hypocotyl & $* * * *$ \\
Melilotus alba Desr. & 1.93 & 7.48 & $* * * *$ \\
Melilotus indicus L. & 6.77 & 30.73 & $* * * *$ \\
Malva parviflora L. & 9.67 & 41.53 & $* * *$ \\
Solanum nigrum L. & 10.81 & 49.85 & $* * *$ \\
Nasturtium officinale W.T. & 13.06 & 61.58 & $* *$ \\
Urtica dioica L. & 22.14 & 66.05 & $* *$ \\
Cyperus sp. & 25.68 & 83.58 & $* *$ \\
\hline
\end{tabular}


TABLE 2 (Continued)

\begin{tabular}{|c|c|c|c|}
\hline \multirow{2}{*}{ Species } & \multicolumn{2}{|c|}{ Extension \% } & \multirow{2}{*}{ Criteria } \\
\hline & Radicle & Hypocotyl & \\
\hline Vicia sativa $\mathrm{L}$. & 31.98 & 79.91 & $*$ \\
\hline Medicago parviflora E.H.L. & 39.19 & 83.58 & $*$ \\
\hline Achyranthes aspera L. & 49.10 & 87.98 & \\
\hline Crotalaria medicaginea Lamk. & 49.90 & 89.69 & \\
\hline Amaranthus viridis L. & 52.71 & 113.79 & \\
\hline Xanthium strumarium $\mathrm{L}$. & 52.95 & 113.71 & \\
\hline Phalaris minor Retz. & 53.68 & 70.60 & \\
\hline Avena fatua $\mathrm{L}$. & 57.54 & 93.02 & \\
\hline Oxalis corymbosa DC. & 59.03 & 114.55 & \\
\hline Pentanema divaricatum Cass. & 61.68 & 92.51 & \\
\hline Trichodesma indicum (L.) & 65.76 & 107.14 & \\
\hline Plantago lanceolata $\mathrm{L}$. & 68.50 & 66.31 & \\
\hline Ipomoea cornea Mart. & 68.58 & 115.38 & \\
\hline Lantana camara L. & 69.37 & 122.43 & \\
\hline Salvia moocroftiana Wall. & 70.60 & 81.40 & \\
\hline Cirsium arvense (L.) & 71.83 & 85.71 & \\
\hline Cyperus rotundus $\mathrm{L}$. & 71.91 & 100.47 & \\
\hline Barleria cristata $\mathrm{L}$. & 77.68 & 95.57 & \\
\hline Helianthus annuus L. (Sepals) & 77.68 & 85.63 & \\
\hline Cannabis sativa $\mathrm{L}$. & 78.34 & 117.11 & \\
\hline Phalaris aquatica $\mathrm{L}$. & 81.72 & 127.91 & \\
\hline Anagallis arvensis L. & 82.69 & 122.92 & \\
\hline Conyza bonariensis (L.) & 82.69 & 99.67 & \\
\hline Taverniera cuneifolia Roth. & 83.64 & 99.42 & \\
\hline Sida cordata (Burm.f.) & 84.27 & 105.50 & \\
\hline Centaurea iberica $\mathrm{T}$. & 86.81 & 124.58 & \\
\hline Convolvulus arvensis $\mathrm{L}$. & 87.50 & 149.48 & \\
\hline Saussurea heteromalla D.Don. & 89.14 & 114.98 & \\
\hline Parthenium hysterophorus L. & 89.93 & 116.74 & \\
\hline Carthamus oxyacantha M. & 91.14 & 122.82 & \\
\hline Coriandrum sativum L. & 92.13 & 108.89 & \\
\hline Digera muricata (L.) & 93.69 & 100.77 & \\
\hline Chenopodium ambrosioides Briq. & 93.81 & 110.47 & \\
\hline Taraxacum officinale L. & 94.14 & 85.04 & \\
\hline Commelina benghalensis L. & 94.85 & 98.32 & \\
\hline Micromeria biflora Buch. & 95.21 & 90.00 & \\
\hline Dichanthium annulatum Forssk. & 95.31 & 100.96 & \\
\hline Artemisia scoparia $\mathrm{W} \& \mathrm{~K}$. & 95.72 & 100.00 & \\
\hline Pteris cretica L. & 95.72 & 94.62 & \\
\hline Nerium oleander L. & 96.74 & 109.23 & \\
\hline Scrophularia altaica Murray. & 96.99 & 105.50 & \\
\hline Helianthus annuus L. (petals) & 97.93 & 95.57 & \\
\hline Rhynchosia minima (L.) & 98.15 & 105.05 & \\
\hline Sonchus asper (L.) & 98.65 & 93.84 & \\
\hline Argyrolobium roseum Camb. & 99.29 & 100.00 & \\
\hline Verbena tenuisecta Briq. & 100.10 & 102.99 & \\
\hline
\end{tabular}


TABLE 2 (Continued)

\begin{tabular}{lccc} 
& \multicolumn{2}{c}{ Extension $\%$} & Criteria \\
\multicolumn{1}{c}{ Species } & Radicle & Hypocotyl & 89.69 \\
Saxifraga rotundifolia L. & 100.80 & 106.92 \\
Salvia aegyptiaca L. & 101.83 & 89.18 \\
Otostegia limbata Benth. & 102.46 & 128.21 \\
Sida alba L. & 102.63 & 108.01 \\
Peganum harmala L. & 103.59 & 93.75 \\
Oxalis corniculata L. & 104.13 & 100.92 \\
Euphorbia hirta L. & 104.99 & 118.04 \\
Rumex nepalensis Spreng. & 106.31 & 102.31 \\
Adiantum caperis -veneris L. & 106.42 & 87.65 \\
Typha minima Funck. & 107.14 & 99.67 \\
Oenothera rosea L' Her. & 111.22 & 120.23 \\
Solanum erianthum D Don & 112.61 & 105.61 \\
Potamogeton lucens L. & 117.13 & 95.92 \\
Lythrum salicaria L. & 117.61 & 105.08 \\
Cissampelos pareira L. & 117.65 & 109.76 \\
Aloe vera (L.) & 118.53 & 92.99 \\
Anisomeles indica (L.) & 121.19 & 102.47 \\
Euphorbia helioscopia L. & 233.48 & 115.81 \\
Vernonia anthelmintica L. & 234.24 & \\
\hline & &
\end{tabular}

TABLE 3

Extraction of phytochemical contents of Melilotous indicus

\begin{tabular}{lcc} 
& TP content $(\mu \mathrm{g} \mathrm{GAE} / \mathrm{mg}$ extract $)$ & TF content $(\mu \mathrm{g}$ QE/ mg extract) \\
Methanolic Extract of $M$. indicus & 211.465517241379 & 190.694 \\
\hline
\end{tabular}

$\mathrm{TF}=$ Total flavonoid, $\mathrm{TP}=$ Total phenolic.

of Melilotus indicus. On the basis of standard regression lines for gallic acid $(\mathrm{y}=0.0232 \mathrm{x}$ - 0.0724; $\left.\mathrm{R}^{2}=0.9991\right)$ and quercetin $(\mathrm{y}=$ $\left.0.0252 \mathrm{x}+0.0358 ; \mathrm{R}^{2}=0.9971\right)$, the equivalents of TPC and TFC were calculated (Table 3). $M$. indices showed the maximum quantity of TPC (211.465517241379 $\mu \mathrm{g}$ GAE/g dry sample). Flavonoids were found to be rich in $M$. indicus $190.694 \mu \mathrm{g}$ QE/ mg extract dry sample). The results of the phytochemical analysis of methanolic extracts of $M$. indicus are listed in table 4 (Table 4). Qualitative analysis of $M$. indicus ensured the presence of tannins, phenols,
TABLE 4

Phytochemical analysis of $M$. indicus

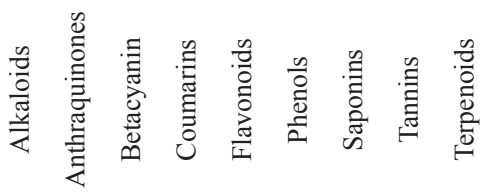

M. indicus $\quad++\quad-\quad++\quad+\quad++++++\quad+\quad++++$

$(+)$ present, $(-)$ absent, $(++)$ moderate concentration, $(+++)$ abundant concentration. 
flavonoids, and coumarins in plant methanolic extracts except anthraquinones were absent.

\section{DISCUSSION}

Extracts from $M$. indicus show fairly good antibacterial and antitumor activities in screening experimentations (Miri, Rad, Alfatemi, \& Rad, 2013). The occurrence of C-glycosides, methylene-dioxypterocarpan, pterocarpans, prenylated pterocarpan and flavone glycoside from this plant have been reported (Yadava \& Jain, 2005; Ali, Shinwari, \& Khan, 2019). Some literature reported that it has allelopathic potential to suppress seedling growth and germination (Gomaa et al., 2014). Previously no allelopathic activities or other ecological information was reported about the second strongly inhibitory Medicago parviflora species. Melilotus alba is found to be the third most inhibitory on lettuce seedling growth. Methanolic extracts of $M$. alba have best antitumor activities. Biochemical compound flavones, volatile oils, resins, and tannins are reported from $M$. alba (Stefanović, Tešić, \& Čomićet, 2015). However, the allelopathic potential of $M$. alba has not been previously reported. $M$. alba in combination with other plants have been used in weeds management.

Coronopus didymus is found topmost strong allelopathic plants during Dish pack analysis. C. didymus commonly known as lesser swinecress native to South America particularly to Brazil is an annual but for some time biannual herb. C. didymus mostly found in disturbing situations such as cultivated land, roadsides, wasteland, winter farming, and uncompetitive pastures. The plant is valued in traditional medicine as a treatment for cancer, gangrene, hemorrhoids, allergies, and wounds. A plant decoction and leaf bandage are used to treat headache and fevers (Khaliq, Hussain, Matloob, Wahid, \& Aslamet, 2013). Extracts of C. didymus has an allelopathic effect on wheat germination and its early seedling growth. Also applied to weed management assessment and extracts of $C$. didymus have allelopathic potential against seed germination of different plants (Marinov-Serafimov, 2010).

The plant allelochemicals have altered interactions among organisms, community dynamics into the soil environment and determine the development of plants in the soil (Meiners, Phipps, Pendergast, Canam, \& Carsonet, 2017). Meanwhile, they affect the cellular structure, metabolism, photosynthesis, enzyme activity, nutrient absorption and hormonal regulation of target plant (Rehman et al., 2019). The extraction of allelochemicals from selective plant species using aqueous or alcoholic solvent comprehends the elaboration of allelopathic effects (de Moraes Gomes et al., 2017). Kasarkar and Barge (2016) applied the aqueous extract of Azadirachta indica leaves on wheat, mung, jowar, and cowpea and observed significant allelopathic effect on shoot length, biomass, root, and germination stage. Anwar, Khalid, Panni, Qureshi, and Rashid (2017) noted the suppression of germination and subsequent growth of A. fatua, $H$. annus, Zea mays and $R$. dentatus by aqueous extract of Euphorbia helioscopia.

Versatile compounds are present in plants which play an important role in pharmaceutical industries for the treatment of various complications and ailments in human as well in animals and plants. The presence of multipurpose phytochemical in plants gets more attention of researchers and physicians these days to deal with the increasing ratio of diseases (Petrovska, 2012). Plants are opulent by having various bio-compounds with diverse polarities (Jones \& Kinghorn, 2006). Methanolic extracts have a large amount of TPC and TFC followed the rule that more compounds are solvable in polar solvents at room temperature (Pin et al., 2010). In present qualitative study of phytochemicals gives evidence of medicinal propensity of the selected weed. The allelochemicals were screened that impart biologically dynamic nature to the plants. The results of this screening analysis confirmed the presence of variant compounds terpenoids, coumarins, flavonoids, tannins, phenols, alkaloids, saponins, and betacyanin. These biologically 
active allelochemicals have antioxidant, antimicrobial, anticancer, antifungal, and antidiabetic medicinal activities. Anti-inflammatory and hypoglycemic activities are reported from Tannins, flavonoids, and saponins while central nervous system activities and analgesic properties with terpenoids (Ullah, Jan, Gul, Khan, \& Sher, 2018).

Treatment of various diseases with natural antioxidants get a worth reputation in this modern era and the traditional herbal medicinal value have been raised. During the assessment of the antioxidant capability of crude plant extract, the aptitude of DPPH radicle scavenging is considered as a milestone. This bioassay evaluation of plants antioxidants capabilities is very low-cost and time-limited (Majid et al., 2015). In this study, M. indicus showed enormous scavenging activity. Good scavenging capabilities may be exhibited by phenolics and flavonoids present in the huge amount due to the donation of electron or hydrogen to stabilize DPPH free radicles. Ullah et al. (2018) reported the antioxidant, analgesic, antimicrobial, anti-angionic, cytostatic and anti-allergic activities of flavonoids. Therefore these observations support the utilization of $M$. indicus in herbal medications and recommended as a potential source of significant allelochemicals for the pharmaceutical industry.

In view of results obtained from quantitative and qualitative analysis various compound have been identified from $M$. indicus methanolic extracts of leaves. The main ingredients present in methanolic extracts were total phenolic content and possible high fungicidal activity is due to the presence of phenolic components. TPC compounds are abundantly found plants secondary metabolites and have about ten thousand individual compounds (Majid et al., 2015). Phenolic or polyphenol can be defined chemically as a substance which possesses a benzene ring with one or more hydroxyl groups, with evidence that increased hydroxylation results in increased toxicity. Plants showed some resistance to microorganism, herbivores and insects are mainly due to these polyphenols. The obtained results of this study were agreed with previously stated that the resistance to phytopathogenic microorganisms is because of the phenolic pathway activation (El-Khateeb et al., 2013). Sisti et al., (2008) also stated the TPC are vigorous to counter microorganisms of pathogenic nature for human beings and animals. Phenolic components counteract envelopment of harmful mediators through several proposed modes of action. These involve in enzymatic processes impairing, weakening the synthesis structural element, rescinding cell membrane permeability barrier, changing the cells physiological eminence and affecting the synthesis of nucleic acids (Bajpai, Sharma, \& Baekt, 2013; Fadli et al., 2012).

The noxious weeds with medicinal properties may have strong allelochemicals potential that can help in the development of bioactive compounds from plant species to be used as natural herbicides for environmentally friendly sustainable control of weeds. $M$. indicus was identified as the strongest allelopathic plant. The result showed that $M$. indicus strongly inhibit the seedling growth of test weed species. Various allelochemicals i.e. TPC, TFC, tannins, phenols, flavonoids and coumarins were identified from the extract of $M$. indicus. There is a dire need to develop a complete database of plants having strong allelopathic potential. It is also recommended to isolate and enlist the allelochemicals present in weeds that's may be used for weed and pest control.

Ethical statement: authors declare that they all agree with this publication and made significant contributions; that there is no conflict of interest of any kind; and that we followed all pertinent ethical and legal procedures and requirements. All financial sources are fully and clearly stated in the acknowledgements section. A signed document has been filed in the journal archives.

\section{ACKNOWLEDGMENTS}

The present study was funded by the Higher Education Commission of Pakistan, 
Islamabad. Developed at the Department of Environmental Science, International Islamic University, Islamabad.

\section{RESUMEN}

Potencial alelopático de 73 especies de malezas en Pakistán. Introducción: El uso de aleloquímicos como sustitutos baratos y amigables con el ambiente para el manejo de hierbas en plantaciones es un tema central actualmente. Objetivo: En el presente estudio se midió la actividad alelopática de 73 hierbas en Pakistán a través de los métodos "sandwich" y "dish pack", con el fin de investigar los compuestos aleloquímicos más importantes en las hierbas analizadas. Métodos: A través del método "sandwich" se determinaron los efectos alelopáticos de los lixiviados de hojas en las hierbas analizadas, mientras que el efecto alelopático volátil a través de la técnica "dish pack". Para investigar el potencial aleloquímico de Melilotus indicus se aplicaron técnicas fitoquímicas tanto cualitativas como cuantitativas. El análisis estadístico de los datos describió el efecto alelopático de 73 especies de hierbas sobre el crecimiento radicular y del hipocótilo en plántulas de lechuga. Resultados: El porcentaje de elongación radicular y del hipocotilo varió entre $0-74 \%$ hasta $0-148 \%$ y $0-75$ $\%$ hasta $0-84 \%$ respectivamente, con una concentración de $10 \mathrm{mg}$ de polvo de planta seca con el método "sandwich". Además, fue entre 2-234 \% y 7-150\% con el método "dish pack". Entre las plantas analizadas para actividad fitotóxica, $M$. indicus presentó los aleloquímicos más fuertes. Los análisis cualitativos y cuantitativos de $M$. indicus mostraron la presencia de flavonoides y compuestos fenólicos, junto con otros aleloquímicos. Conclusiones: Las hierbas seleccionadas pueden tener un potencial aleloquímico fuerte que ayude al desarrollo de compuestos bioactivos de plantas, para usar como hierbicidas y pesticidas naturales en el manejo sostenible de plagas y malas hierbas.

Palabras clave: alelopatía; bioensayo; ELISA tipo sándwich; fitoquímicos.

\section{REFERENCES}

Abbas, T., Nadeem, M. A., Tanveer, A., Ali, H. H., \& Farooq, N. (2018). Role of allelopathic crop mulches and reduced doses of tank-mixed herbicides in managing herbicide-resistant Phalaris minor in wheat. Crop Protection, 110, 245-250.

Ali, K. W., Shinwari, M. I., \& Khan, S. (2019). Screening of 196 medicinal plant species leaf litter for allelopathic potential. Pakistan Journal of Botany, 51(6), 2169-2177.

Ali, S., Tahir, M. N., Shah, G. A., Khan, M. A., \& Mehmood, S. (2017). Dynamics of seed bank and weed flora in rain-fed wheat as affected by different tillage systems. Pakistan Journal of Agricultural Sciences, 54(4), 907-917.

Anwar, T., Ilyas, N., Qureshi, R., Qureshi, H., Khan, S., Khan, S. A., Fatimah, H., \& Waseem, M. (2019). Natural herbicidal potential of selected plants on germination and seedling growth of weeds. Applied Ecology and Environmental Research, 17(4), 9679-9689.

Anwar, T., Khalid, S., Panni, M. K., Qureshi, H., \& Rashid, M. (2017). Allelopathic effect of Euphorbia helioscopia on Avena fatua, Rumex dentatus, Helianthus annuus, Zea mays and Triticum aestivum. Pakistan Journal of Weed Science Research, 23(2), 165-177.

Appiah, K., Li, Z., Zeng, R. S., Luo, S., Oikawa, Y., \& Fujii, Y. (2015). Determination of allelopathic potentials in plant species in sino-japanese floristic region by sandwich method and dish pack method. International Journal of Basic and Applied Sciences, 4(4), 381-394.

Bajpai, V. K., Sharma, A., \& Baek, K. H. (2013). Antibacterial mode of action of Cudrania tricuspidata fruit essential oil, affecting membrane permeability and surface characteristics of food-borne pathogens. Food Control, 32(2), 582-590.

Bhadoria, P. (2011). Allelopathy: A natural way towards weed management. American Journal of Experimental Agriculture, 1(1), 7-20.

de Moraes Gomes, M., Bertoncelli, D. J., Alves, G. A. C., Freiria, G. H., Furlan, F. F., Gomes, G. R., Favoretto, V. R., Neto, H. F. I., Omura, M. S., \& de Souza, J. R. P. (2017). Allelopathic potential of the aqueous extract of Raphanus sativus L. on the germination of beans and corn seeds. Open Access Library Journal, 4, 1-10.

El-Khateeb, A. Y., Elsherbiny, E. A., Tadros, L. K., Ali, S. M., \& Hamed, H. B. (2013). Phytochemical analysis and antifungal activity of fruit leaves extracts on the mycelial growth of fungal plant pathogens. Journal of Plant Pathology and Microbiology, 4, 1-6.

Fadli, M., Saad, A., Sayadi, S., Chevalier, J., Mezrioui, N. E., Pagès, J. M., \& Hassani, L. (2012). Antibacterial activity of Thymus maroccanus and Thymus broussonetii essential oils against nosocomial infection-bacteria and their synergistic potential with antibiotics. Phytomedicine, 19(5), 464-471.

Fisher, M. C., Henk, D. A., Briggs, C. J., Brownstein, J. S., Madoff, L. C., McCraw, S. L., \& Gurr, S. J. (2012). Emerging fungal threats to animal, plant and ecosystem health. Nature, 484(7393), 186-194.

Fujii, Y. (2005). Dish pack method: a new bioassay for volatile allelopathy. Thymus, 2(3), 493-497.

Fujii, Y., Shibuya, T., Nakata, K., Itani, T., Hiradate, S., \& Parvez, M. M. (2004). Assessment Method for the 
Allelopathic Effect from Leaf Leachate. Weed Biology Management, 4, 19-23.

Gomaa, N. H., Hassan, M. O., Fahmy, G. M., González, L., Hammouda, O., \& Atteya, A. M. (2014). Allelopathic effects of Sonchus oleraceus L. on the germination and seedling growth of crop and weed species. Acta Botanica Brasilica, 28(3), 408-416.

Jones, W. P., \& Kinghorn, A. D. (2012). Extraction of plant secondary metabolites. In S. D. Sarker, Z. Latif, \& A. I. Gray (Eds.), Natural products isolation (pp. 323351). EE.UU: Humana Press.

Kasarkar, A. R. \& Barge, A. N. (2016). Effect of aqueous extract of neem (Azadirachta indica A. Juss) leaves on germination and growth of some agricultural crops. Journal of Medicinal Plants, 4(5), 11-13.

Khaliq, A., Hussain, S., Matloob, A., Wahid, A., \& Aslam, F. (2013). Aqeous swine cress (Coronopus didymus) extracts inhibit wheat germination and early seedling growth. International Journal of Agriculture and Biology, 15(4), 743-748.

Khan, S., Shinwari, M. I., Haq, A., Ali, K. W., Rana, T., Badshah, M., \& Khan, S. A. (2018). Fourier-transform infrared spectroscopy analysis antifungal activity of methanolic extracts of Medicago parviflora, Solanum nigrum, Melilotus alba and Melilotus indicus On Soil-Borne Phytopathogenic Fungi. Pakistan Journal of Botany, 50(4), 1591-1598.

Kim, D. O., Jeong, S. W., \& Lee, C. Y. (2003). Antioxidant capacity of phenolic phytochemicals from various cultivars of plums. Food Chemistry, 81(3), 321-326.

Majid, M., Khan, M. R., Shah, N. A., Haq, I. U., Farooq, M. A., Ullah, S., \& Sajid, M. (2015). Studies on phytochemical, antioxidant, anti-inflammatory and analgesic activities of Euphorbia dracunculoides. BMC Complementary and Alternative Medicine, $15(1), 349-362$.

Marinov-Serafimov, P. (2010). Determination of allelopathic effect of some invasive weed species on germination and initial development of grain legume crops. Pesticides and. Phytomedicine, 25(3), 251-259.

Meiners, S. J., Phipps, K. K., Pendergast, T. H., Canam, T., \& Carson, W. P. (2017). Soil microbial communities alter leaf chemistry and influence allelopathic potential among coexisting plant species. Oecologia 183(4), 1155-1165.

Miri, A., Rad, J. S., Alfatemi, S. M. H., \& Rad, M. S (2013). A study of antibacterial potentiality of some plants extracts against multidrug resistant human pathogens. Annals of Biological Research, 4(8), $35-41$
Park, Y. S., Jung, S. T., Kang, S. G., Heo, B. G., Arancibia-Avila, P., Toledo, F., \& Gorinstein, S. (2008). Antioxidants and proteins in ethylene-treated kiwifruits. Food Chemistry, 107(2), 640-648.

Petrovska, B. B. (2012). Historical review of medicinal plants' usage. Pharmacognosy Reviews, 6(11), 1.

Pin, K. Y., Chuah, A. L., Rashih, A. A., Mazura, M. P., Fadzureena, J., Vimala, S., \& Rasadah, M. A. (2010). Antioxidant and anti-inflammatory activities of extracts of betel leaves (Piper betle) from solvents with different polarities. Journal of Tropical Forest Science, 22(4), 448-455.

Rehman, S., Shahzad, B., Bajwa, A. A., Hussain, S., Rehman, A., Cheema, S. A., ... Li, P. (2019). Utilizing the allelopathic potential of Brassica species for sustainable crop production: a review. Journal of Plant Growth Regulation, 38(1), 343-356.

Richardson, P. M. (1990). Phytochemical Methods: A Guide to Modern Techniques of Plant Analysis. Brittonia, 42(2), 115-115.

Shinwari, M. I., \& Fujii, Y. (2013). Allelopathic activity of medicinal plants and weeds from Pakistan. Allelopathy Journal, 32(2), 223-232.

Shinwari, M. I., Shinwari, M. I., \& Fujii, Y. (2013). Allelopathic evaluation of shared invasive plants and weeds of Pakistan and Japan for environmental risk assessment. Pakistan Journal of Botany, 45(1), 467-474.

Sisti, M., De Santi, M., Fraternale, D., Ninfali, P., Scoccianti, V., \& Brandi, G. (2008). Antifungal activity of Rubus ulmifolius Schott standardized in vitro culture. LWT-Food Science and Technology, 41(5), 946-950.

Stefanović, O. D., Tešić, J. D., \& Čomić, L. R. (2015). Melilotus albus and Dorycnium herbaceum extracts as source of phenolic compounds and their antimicrobial, antibiofilm, and antioxidant potentials. Journal of Food and Drug Analysis, 23(3), 417-424.

Trease, G. E., \& Evans, W. C. (1989). Phytochemical screening. Pharmacognsy (11th edn.). Brailliar Tiridel Can.

Ullah, S., Jan, G., Gul, F., Khan, S., \& Sher, J. (2018). Antifungal, nutritional and phytochemical investigation of Asplenium dalhousiae of district Dir Lower, Pakistan. Journal of Pharmacognosy and Phytochemistry, 7(2), 3281-3288.

Yadava, R. N., \& Jain, S. (2005). A new bioactive flavone glycoside from the seeds of Melilotus indica All. Journal of Asian Natural Products Research, 7(4), 595-599. 